\title{
Teorías Implícitas y Esencialismo Psicológico: Herramientas Conceptuales Para el Estudio de las Relaciones Entre y Dentro de los Grupos
}

\section{Implicit Theories and Psychological Essentialism: Conceptual Tools for the Study of the Relations Between and Within the Groups}

\author{
Claudia Estrada y Miriam Oyarzún \\ Universidad de Magallanes
}

\author{
Vincent Yzerbyt \\ Université catholique de Louvain
}

\begin{abstract}
Dentro de la psicología social, las teorías implícitas constituyen un campo reciente de estudio que se ha revelado, en las últimas décadas, como un ámbito promisorio de investigación para la comprensión de fenómenos grupales. El presente artículo se ocupa de las teorías implícitas que conciernen a la naturaleza de los grupos humanos, y en particular al esencialismo psicológico. El esencialismo es la tendencia a creer que los objetos tienen una esencia o naturaleza subyacente que hace que sean lo que son. Nuestra revisión de investigaciones recientes indica que el esencialismo es una variable interesante de estudiar ya que afecta a los fenómenos que son vulnerables a la percepción social tales como la explicación de las diferencias, la desviación intragrupal y la proyección social.
\end{abstract}

Palabras Clave: psicología social, esencialismo, teorías implícitas, relaciones intergrupo, relaciones intragrupo.

\begin{abstract}
In social psychology, the implicit theories are a recent field of study. Nevertheless, in the last decades, they have been revealed as a promissory area of investigation for the understanding of group's phenomena. The present paper gives attention to the implicit theories that concern the nature of human groups and, in particular psychological essentialism. Essentialism is the belief that an underlying essence lies at the root of every social category. Our revision of some recent investigations indicates that the essentialism is an interesting variable to consider because it has impact in all phenomena that are vulnerable to social perception such as the in-group deviation and the social projection.
\end{abstract}

Keywords: social psychology, essentialism, implicit theories, inter-group relations, intra-group relations.

Un niño de origen chileno es adoptado por una familia finlandesa a la edad de 6 meses. A sus 15 años y consciente de ser un hijo por adopción, no conoce nada de su pasado ni de la historia ni la cultura de sus padres biológicos. ¿Se considerará a sí mismo como chileno, finlandés o una mezcla de ambos? Y sus padres biológicos y adoptivos ¿qué contestarían? La respuesta a estas preguntas depende de la teoría implícita sobre la naturaleza de los

Claudia Estrada Goic, Facultad de Humanidades, Ciencias Sociales y Ciencias de la Salud, Universidad de Magallanes, Chile.

Vincent Yzerbyt, Departamento de Psicología Social y Organizacional, Université catholique de Louvain, Bélgica. Miriam Oyarzún Jara, Dirección de Asuntos Estudiantiles. Universidad de Magallanes, Chile.

La correspondencia relativa a este artículo deberá ser dirigida a Claudia Estrada, Av. Bulnes 01855, 6200000 - Punta Arenas, Chile. E-mail: claudia.estrada@umag.cl

Esta investigación fue financiada con fondos del Proyecto de Investigación Interna UMAG 025600 y se enmarca en el Proyecto FONDECYT 11060001 "Proyección social: El rol moderador del esencialismo". grupos sociales que cada uno cultive: si creen que las personas poseen una esencia interior que define lo que ellos son o, por el contrario, son el reflejo de las oportunidades, experiencias y elecciones que han tenido.

Aunque el estudio de las teorías implícitas constituye un campo de interés científico bastante temprano en la psicología social (Bruner \& Tagiuri, 1954; Cronbach, 1955; Gross, 1961; Kelly, 1955; Newcomb, 1931; Thorndike, 1920; Wishner, 1960), éste ha aumentado a partir de los años ochenta (Beauvois, 1984; Beauvois \& Joule, 1982; Leyens, 1983). La importancia de esta área de investigación radica en que las teorías implícitas participan en numerosos procesos psicosociales. Estudios recientes realizados sobre la formación de impresión y las estrategias cognitivas de aprehensión de la realidad social, han dado un impulso importante al concepto de teoría implícita (Castano, 2004; Estrada, Yzerbyt \& Seron; 2004; Hong, Chiu, Dwek, Lin \&Wan, 1999; Levy, Chi-yue \& Yingyi, 2006; Nisbett, Peng, Choi \& Norezayan, 2001; Plaks, Stroessner, Dweck \& Sherman, 2001; entre otros). El 
propósito de este artículo es brindar una revisión teórica sobre los conceptos de teoría implícita y el esencialismo psicológico, ligado al tema de las relaciones intergrupales.

\section{¿Qué son las Teorías Implícitas?}

Teoría Implícita (TI, en adelante) se refiere a un conjunto de creencias que un individuo posee respecto a cómo son las personas, la naturaleza humana y/o los grupos sociales. Estos conocimientos se organizan de modo más o menos coherente y establecen relaciones entre los diferentes aspectos de la realidad. El término teoría hace referencia a que son un conjunto relativamente consistente de creencias que sirven para predecir un cierto número de fenómenos. Aunque algunos autores, tales como Rodrigo (1994), han planteado que el término teoría no es el más adecuado ya que se entiende como un cuerpo de preposiciones, a la fecha no han surgido nuevas expresiones más precisas que permitan reemplazarlo. Implícito, por su parte, describe la idea de que estas creencias no se basan en teorías formales y que las personas no son necesariamente conscientes del impacto de éstas sobre su comportamiento (Leyens, 1983). Un concepto afín al de TI es el de representaciones sociales, que puede ser definido como aquellos esquemas de conocimiento compartidos acerca de la realidad social que orientan la conducta de las personas de un grupo social. La representación social es un concepto más amplio que el de TI ya que involucra una serie de procesos tales como la difusión y asimilación de conocimientos, el desarrollo individual y colectivo, la definición de la identidad personal, la expresión de grupo y las transformaciones sociales. Ambos conceptos son comparables en que poseen cargas motivacionales y afectivas que incitan a la acción (Rodrigo, 1994).

Las teorías implícitas también han sido llamadas "teorías ingenuas" o "de sentido común", haciendo referencia a la dicotomía existente entre el conocimiento científico y el que no lo es. Furnham (1990) sugiere que las TI difieren de las científicas en varias dimensiones específicas. Según este autor, las teorías científicas son explícitas, formales, coherentes y consistentes, deductivas y rara vez confunden las causas con los efectos; además se centran en los procesos y tienden a ser más específicas que generales. Las TI por su parte, son descritas como más informales, poco consistentes, buscan un conocimiento confirmatorio mediante la verificación de las creencias, son inductivas, tienden a confundir las causas y las consecuencias, y son más bien generales que específicas (ver también Rodrigo, Rodríguez \& Marrero, 1993).

Según Bacova (1998), las TI influencian la forma en que cada persona concibe la realidad y son susceptibles de ser particulares para cada dominio del mundo social. Por ejemplo, no existe necesariamente interdependencia entre las teorías sobre la inteligencia, la etnia, el género o los principios morales.

\section{Funciones de las Teorías Implícitas}

Las teorías implícitas tienen diversas funciones que han sido resumidas por Levy y su equipo (Levy, et al., 2006):

1. Sirven para comprender y simplificar la realidad social, aumentando la percepción de control psicológico en nuestras relaciones sociales.

2. Nos permiten organizar la realidad social, asignando etiquetas a las observaciones y experiencias que vivimos cotidianamente.

3. Nos ayudan a compartir el "sentido común", que reúne y sintetiza nuestros valores y formas de interpretación de la realidad.

4. Sirven para proteger nuestro autoconcepto y el de nuestro grupo, ya que otorgan un marco justificativo para las actitudes intergrupo y las desigualdades sociales.

\section{Tipos de Teorías Implícitas}

Las TI pueden ser agrupadas en tres grandes categorías: las TI sobre la personalidad, las TI respecto de la naturaleza humana, y las TI que hacen referencia a la naturaleza u origen de los grupos.

Teorías implícitas sobre la personalidad (TIP, en adelante). Fueron definidas la primera vez por Bruner y Tagiuri (1954, p. 642) como "el conocimiento que tenemos sobre una persona y la forma en que utilizamos este conocimiento para realizar inferencias sobre su personalidad". Numerosos estudios han intentado comprender el proceso mediante el cual nos formamos impresiones globales sobre las personas que conocemos recientemente, usando nuestros conocimientos o teorías generales de cómo son las personas. La existencia de TIP parece responder a nuestra necesidad de "conocer", "controlar" y "predecir" el comportamiento de quienes se encuentran en nuestro ambiente social. Las TIP dependen de los individuos que las contienen, ya que las personas están atentas a los rasgos que son relevantes para su autoconcepto (Kelly, 1955; Markus, 1977). A 
pesar de este componente individual contenido en cada TIP, la gran mayoría de estas teorías posee elementos comunes que son compartidos por un gran número de personas (Chiu, Hong \& Dweck, 1997; Secord \& Backman, 1974). Por ejemplo, aunque cada individuo le otorgue elementos y explicaciones únicas a la creencia de que las personas extrovertidas son más cálidas que las introvertidas, todas ellas comparten aspectos generales.

Teorías implícitas sobre la naturaleza humana. Pueden ser definidas como las creencias generales que cada persona posee sobre el ser humano. Según Leyens (1983), estas teorías son un caso general de TI en el que se incluyen todas las teorías sobre la bondad o maldad de la raza humana, sobre su maleabilidad, su forma de ser, etc.

Dentro de las TI sobre la naturaleza encontramos las teorías sobre el locus de control y las teorías sobre la naturaleza humana propiamente tal.

La teoría del locus de control (Rotter, 1966), considerada como una de las primeras teorías explicativas de la atribución social, ha sido también entendida como una TI porque hace referencia a la forma como las personas nos representamos la naturaleza humana y los determinantes de su comportamiento (Morris, Menon \& Ames, 2001). Basándose en los postulados de Heider (1958), Rotter (1966) plantea que una persona puede estimar que su comportamiento es provocado, ya sea por factores internos (locus de control interno) o por factores externos a sí mismo (locus de control externo). Abundantes investigaciones han mostrado que esta TI es pertinente en el estudio de diversos fenómenos tales como la resistencia al conformismo, el éxito académico, la ansiedad y la reacción frente al fracaso (Lefcourt, 1973). Más recientemente ha sido estudiada en relación al comportamiento de riesgo (Hunter, 2002; Miller \& Mulligan, 2002), la satisfacción laboral (Kirkcaldy, Shephard \& Furnham, 2002), la adaptación escolar (Manger, Eikeland \& Asbjornsen, 2002) y al desorden alimentario (Fouts \& Vaughan, 2002).

Dweck y sus colegas (ver Dweck, Chiu \& Hong, 1995; Dweck, Hong \& Chiu, 1993, para una revisión sobre el tema) propusieron un modelo que explica la forma en la que las personas construyen teorías respecto de ciertos aspectos de la naturaleza humana. El modelo identifica dos TI que se aplican a la inteligencia, el carácter moral, la personalidad y, en general, a la concepción del mundo. Se trata de la teoría implícita "entitativista" y la teoría implícita "incrementalista". Quienes emplean la primera creen que las características de una persona se organizan en torno a un núcleo central relativamente fijo y no maleable. Numerosas investigaciones han señalado que estos teóricos "entitativistas" tienden a realizar juicios más definitivos, más causales $\mathrm{y}$, a partir de informaciones parciales, dicotomizan la información negativa y positiva, estableciendo juicios diagnósticos a partir de un proceso de inferencia y realizando atribuciones disposicionales.

Los teóricos "incrementalistas", por su parte, creen que la configuración general de una persona es relativamente maleable y susceptible de un desarrollo continuo. En general, tienden a realizar juicios en términos de proceso y no de causa-efecto, integran la información positiva y negativa, utilizan procesos mediacionales para comprender el comportamiento de los otros, y prestan atención a la influencia de los factores situacionales (Dweck et al., 1995; Dweck et al., 1993; Hong, Chiu, Dweck \& Sacks, 1997). Aunque ambas teorías parecen ser mutuamente excluyentes sobre un plan teórico, la manipulación experimental ha mostrado que los individuos pueden cambiar de posición sobre un continuo (Dweck et al., 1995).

Los estudios sobre los efectos de estas TI sobre los niveles personales e interpersonales indican que éstas tienen impacto en la forma en que se explica el rendimiento intelectual (Dweck et al., 1995; Hong et al., 1999); la orientación hacia los "derechos" y "deberes" (Chiu et al., 1997); la evaluación de la trasgresión y la asignación de castigo (Gervey, Chiu, Hong \& Dweck, 1999); y el manejo de la información inconsistente (Chiu, Dweck, Tong \& Fu, 1997). Respecto de sus efectos sobre el nivel social, los resultados muestran que los teóricos "entitativistas" adhieren más a los estereotipos cuando estos parecen reflejar diferencias innatas entre los grupos, son más extremos en sus apreciaciones y prefieren la información estereotípica (Levy, Stroessner \& Dweck, 1998; Plaks et al., 2001).

Teorías implícitas sobre la naturaleza de los grupos sociales. Pueden ser definidas como aquellas teorías ingenuas que reagrupan nuestras creencias y conocimientos generales respecto a las condiciones de pertenencia de una persona a un grupo social dado. Ya que el esencialismo es la teoría implícita que nos interesa en el presente artículo, la presentaremos más detalladamente. 


\section{El Concepto de Esencialismo}

El esencialismo es un concepto que ha interesado en el tiempo a diferentes disciplinas. El esencialismo filosófico, desarrollado en el seno de la filosofía, recubre significados diversos desde Aristóteles, Platón pasando por San Agustín y hasta Decartes, Kant, Hegel y Husserl, entre otros (Jerphagnon, 1987). Su principal característica es que utiliza el concepto de esencia para comprender el sentido de la existencia humana. El esencialismo biológico, que nace durante el siglo XVII, establece la existencia de esencias únicas para cada especie, $\mathrm{y}$ se encuentra a la base de la concepción creacionista (ver Evans, 2001). El "esencialismo genético" (Nelkin \& Lindee, 1995) hace referencia a la idea según la cual el hombre puede ser reducido a su composición genética (genoma). El esencialismo étnico, por su parte, nace en el seno de la antropología y se interesa en el por qué las etnias se ven a sí mismas y a otros grupos como dotados de una esencia (GilWhite, 2001).

\section{El Esencialismo Psicológico}

La investigación sobre la percepción grupal muestra que las personas son susceptibles de recurrir a TI para explicar la existencia de los grupos y categorías sociales. La tendencia de los individuos a creer que ciertos grupos poseen una naturaleza biológica o esencial parece ser un fenómeno bastante extenso (Haslam, Rothschild \& Ernst, 2002; Hirschfeld, 1995a, 1995b; Rothbart \& Taylor, 1992; Yzerbyt, Corneille \& Estrada, 2001; Yzerbyt, Rocher \& Schadron, 1997).

Sherif (1948) observó que algunas personas describen los acontecimientos sociales en términos de "esencia", entendiendo que ésta incluye las cualidades inherentes a un grupo. El término "esencialismo" fue utilizado por primera vez por Allport (1954) en su tratado clásico sobre el prejuicio. La creencia en la existencia de una esencia sería el resultado del "principio del menor esfuerzo", según el cual los observadores perciben al grupo social como dotado de componentes comunes a todos sus miembros. Olvidado por la psicología social, el término fue reintroducido en la investigación psicológica por Medin (1989). Según este autor, la similitud perceptiva se basa en representaciones de características de superficie (observables) que son conectadas a características profundas (no observables a simple vista). Definió el esencialismo como "la tendencia de las personas a asignar a los objetos o a pensar que los objetos tienen una esencia o una naturaleza subyacente que hace que sean lo que son" (Medin, 1989, p. 1476). El esencialismo es más probable cuando se trata de similitudes que son posibles de conectar con una sustancia, un código genético, un potencial innato o una estructura molecular. Las consecuencias de la categorización esencialista son que las similitudes de superficie son tratadas como heurísticos, que permiten conocer las propiedades profundas limitando la composición de nuestras representaciones mentales sobre la categoría y sus miembros.

Rothbart y Taylor (1992) importan la noción de esencialismo hacia la psicología social con el propósito de comprender el peso y la influencia que tienen las etiquetas categoriales en materia de categorías sociales. Estos autores distinguen dos grandes tipos de categorías: las naturales y las artificiales. Mientras el primer tipo nos conecta con lo que observamos en el mundo animal, el segundo concierne a los objetos construidos con fines precisos (como autos y lápices). La tendencia de los observadores a comprender las categorías sociales como si ellas fueran naturales comporta su esencialización. Aunque las categorías sociales son más cercanas de las categorías artificiales que las naturales, ellas son dotadas de una especie de esencia natural. Los individuos tienden a considerar la pertenencia a una categoría social como el reflejo de una identidad única y real. Aunque las categorías puedan ser el resultado de las elecciones de los individuos o impuestas por las circunstancias, son dotadas de características observadas en las categorías naturales tales como el potencial inductivo, que consiste en la posibilidad de prever nuevas características a partir de las conocidas, y la inalterabilidad, que consiste en que la categoría a la que se pertenece es estable en el tiempo.

Apoyándose en las proposiciones teóricas de Rothbart y Taylor (1992), Yzerbyt y Schadron (1996) propusieron una descripción del fenómeno del esencialismo psicológico que llamaron "síndrome esencialista". Según estos autores, existen una serie de características que permiten pensar que nos encontramos frente a la "esencialización" de un grupo. Sus principales características son:

1. Estatus ontológico específico: el grupo es percibido como una entidad, como una unidad que comparte un destino común.

2. Inalterabilidad de la pertenencia categorial: si una persona pertenece a un grupo determinado no puede pertenecer a otro. 
3. Carácter inductivo: una vez que conocemos la pertenencia de alguien a un grupo o categoría social, creemos saber más ampliamente sobre él.

4. Fuertes conexiones entre los atributos de una categoría: los atributos se organizan de forma coherente y son interpretados a partir de esta temática unificadora. Se asume la existencia de una relación causal entre la posesión de la esencia y de todas las características del grupo social.

5. Exclusión de otras formas de conocimiento y aprehensión del individuo.

A partir de lo anteriormente señalado en el contexto del esencialismo, la esencia puede ser definida como el conjunto de características y propiedades biológicas, culturales o sociales que se encuentran a la base de un grupo social. La esencia constituye el núcleo estable compartido por los miembros de un mismo grupo, siendo ella la condición necesaria y suficiente para pertenecer a una categoría social. De acuerdo a Estrada (2003), sus características son:

1. La esencia preexiste al grupo. Es su existencia en cada miembro de un grupo lo que ha predispuesto su encuentro y la conformación del grupo.

2. La esencia es invisible pero puede ser inferida. La esencia es por definición no observable directamente. La única posibilidad de conocerla es a través de su manifestación fenotípica (de superficie). Una excepción a esta regla es el gen que puede ser considerado como una representación parcialmente "visible" de la esencia biológica.

3. La esencia es inmutable. La esencia es la parte más estable de un grupo. Aunque existen situaciones de cambio individual que pueden afectar a una parte de la esencia de una persona, esta situación no modifica la esencia del grupo. Por ejemplo, un accidente grave puede modificar las características importantes de un individuo y cambiar su "esencia" para asimilarla a la de su nueva discapacidad.

4. La esencia es responsable de las características de superficie de un grupo. Los comportamientos, las actitudes, los sentimientos, las normas, etc., son expresiones de la esencia del grupo. La esencia no es responsable de todas las características observables en los miembros de un grupo sino únicamente de aquellas que son compartidas por ellos.

5. La esencia es exclusiva. Si un grupo posee una esencia no puede ni cambiarla ni tener más de una al mismo tiempo.

6. La esencia es compartida por todos los miembros de un grupo en el tiempo y el espacio. La esencia es compartida por los miembros de un grupo de forma tal que si me encuentro frente a un comportamiento individual que puede ser la expresión de la esencia de esa persona, puedo extrapolar esa información a todos los miembros del grupo.

7. La esencia se transmite. La esencia no puede elegirse. Es el resultado de la herencia biológica, cultural o social que hemos recibido.

8. La esencia es coherente y consistente. La esencia tiene un potencial de desarrollo con un contenido limitado. El contenido es coherente y consistente de manera tal que si conocemos una parte podemos inferir otra. Esta propiedad le confiere un fuerte poder inductivo.

9. La esencia guarda la potencialidad de desarrollo de un grupo. La esencia determina los rangos de variabilidad de los aspectos contenidos en ella. Las investigaciones realizadas por Yzerbyt y colegas (Estrada, et al., 2004; Yzerbyt et al., 2001; Yzerbyt et al., 1997; Yzerbt \& Rogier, 2001) muestran que el esencialismo es tanto una característica atribuible a categorías y grupos sociales con orígenes "objetivos" (raza, género, genes) como una creencia individual (existen individuos más y menos esencialistas respecto de una determinada categoría social), que puede ser medida tanto como una variable natural (creencia preexistente al estudio que puede ser evaluada mediante un cuestionario de creencias esencialistas) como experimental (puede ser activada mediante manipulación experimental).

\section{Las Causas del Esencialismo}

Diversas perspectivas surgen en la literatura respecto de las razones y formas de funcionamiento de las creencias esencialistas.

Rothbart y Taylor (1992) pensaban que el esencialismo se origina en un "error ontológico" y emerge cuando las personas cometen el error de comprender las categorías sociales (artificiales) como si ellas fueran asimilables a las categorías naturales. Este error induciría a creer por ejemplo, que las diferencias entre dos categorías sociales como judíos y cristianos son tan estables como las diferencias que se pueden observar entre un mineral y un vegetal.

Hirschfeld (1995a; 1995b; 1998; 1999) condujo numerosas investigaciones que le permiten postular que las personas se encuentran equipadas de manera innata de un sistema conceptual responsable de la creación y organización de un "reino humano". Este sistema especializado tiene la facultad de 
clasificar a los seres humanos utilizando diferencias superficiales, pero no incluye la creencia de la existencia de características profundas que serían compartidas por los miembros de una categoría. De esta forma, aunque existen categorías que tienden a ser esencializadas (como la raza), este fenómeno ocurre durante el desarrollo de las representaciones culturales sobre esta categoría, que son reforzadas por el ambiente del niño y no por causa de este sistema innato. Según Hirschfeld (1995b), los niños pequeños ya son capaces de distinguir entre las causas biológicas y sociales así como la forma en la que las primeras son mediatizadas por las segundas. Para este autor, la noción de "esencia" es fruto de un contexto relacional que se instala en un sistema cognitivo innato.

\section{Efectos del Esencialismo en las Relaciones Intra e Intergrupo}

Un interés fundamental de la psicología social es el conocimiento acerca de cómo nos relacionamos unos con otros. La interacción con otros es fundamental para el desarrollo social de los seres humanos aunque los sentimientos y acciones que acompañan este proceso pueden ser tanto positivos como negativos (Myers, 2004). Son numerosos los esfuerzos que se han llevado a cabo para comprender cómo surgen y se mantienen fenómenos tales como el prejuicio, la agresión, el conflicto u otros positivos como el altruismo y la atracción interpersonal, aunque subsisten preguntas respecto a cuáles son las variables mediadoras de estos procesos.

El esencialismo es una teoría implícita que concierne directamente con la organización del ambiente social, por lo que son variados los estudios que se han ocupado de su impacto en fenómenos intra e intergrupo. Uno de los fenómenos estudiados se refiere a la construcción de TI para explicar las diferencias entre los grupos. Hollander y Howard (2000) sugieren que las diferencias intergrupales tienden a ser explicadas por la presencia de naturalezas esenciales. Este fenómeno es particularmente intenso cuando se trata de evaluar diferencias asociadas a lo biológico tales como la "raza", el "género" o la "sexualidad", que son percibidas más como características individuales que como el resultado de la organización de un sistema social. Categorías sociales tales como la raza nos parecen naturales, sin que cuestionemos el que su base "objetiva" (genética) sea tan válida como las diferencias entre los tipos de orejas o de pies; sin embargo, estas características no han sido usadas ni interpretadas de la misma forma.

Martín y Parker (1995) mostraron que cuando explicamos las diferencias entre los géneros y las razas, usamos tanto factores biológicos como sociales. Según estos autores, las personas que prefieren vivir en ambientes claros y definidos (baja tolerancia a la ambigüedad), tienden a utilizar más los factores biológicos. Se piensa que este resultado obedece a que estas personas prefieren usar explicaciones que se basan en causas estables e inmutables. En un estudio reciente, Estrada et al. (2004) investigaron el efecto del esencialismo sobre la explicación de las diferencias grupales. Los resultados indican que el esencialismo modula el uso de factores explicativos biológicos y culturales. Los participantes esencialistas utilizaron más el factor biológico para explicar las diferencias intergrupales, pero no para dar cuenta de las diferencias al interior de su propio grupo. Los avances en esta temática parecen indicar que el esencialismo puede explicar, en parte, las dificultades al momento de integrar incluso pequeñas diferencias entre los grupos, que bajo esta TI son percibidas como irreconciliables.

Otra de las manifestaciones del esencialismo en las relaciones intergrupo es el fenómeno de la infrahumanización. Leyens y sus colegas (Leyens et al., 2000; Leyens et al., 2001; Vaes, Paladino \& Leyens, 2002) se han interesado en cómo se define la esencia humana y en qué circunstancias ésta es o no atribuida a un determinado grupo. Sus resultados indican que las personas explican las diferencias entre los grupos por la existencia de algunas esencias que pueden ser superiores a otras (culturales, religiosas, lingüísticas, etc.). Como los individuos tienden al egocentrismo, asignan a su grupo su propia esencia, humana y superior. Ya que esta esencia es característica del propio grupo, el exogrupo, que posee una esencia diferente, necesariamente se verá dotada de una esencia menos humana o infra humana, e inferior. Este fenómeno ha sido denominado infrahumanización. Los numerosos estudios conducidos por este equipo de investigadores han mostrado consistentemente que las personas niegan la existencia de sentimientos (característica típicamente humana a diferencia de las emociones) en personas que pertenecen a grupos sociales diferentes (Leyens et al., 2000). Bar-Tal (1990), por su parte postula que este fenómeno nos puede llevar a percibir a una persona o grupo como ilegítimo (por ser menos humano), despertando hacia ellos emociones negativas 
de rechazo y miedo. Su falta de humanidad lo convertiría en un peligro para el endogrupo lo que podría potenciar la activación de situaciones de conflicto que pueden derivar en fenómenos tales como el ataque preventivo y otros similares de gran carga destructiva. Después de todo, una persona tiene el legítimo derecho a defenderse de otra considerada como un bárbaro o un infrahumano.

Otro de los aspectos que han interesado a los investigadores ha sido el efecto del esencialismo sobre los estereotipos y el prejuicio. Este tema fue abordado preliminarmente por Allport (1954), quien postuló que la creencia en la existencia de esencias en los grupos es uno de los aspectos de la percepción social ligada directamente al prejuicio. Esta idea fue apoyada empíricamente por Haslam y su equipo (Haslam et al., 2002). Yzerbyt y sus colegas (Rocher, 1997; Yzerbyt et al., 1997) estudiaron la relación entre el esencialismo y los estereotipos, y postularon que un estereotipo contendría no sólo expectativas sobre las características del miembro de un grupo, sino que también una serie de creencias que explican el lazo entre esas características y la naturaleza del grupo. Como resultado, el estereotipo no sirve sólo para reducir el número de informaciones que deben ser integradas al momento de enfrentar a un miembro de un grupo, sino que también permiten inferir características nuevas no observadas. Cuando una categoría social es percibida de una forma esencialista, una serie de TI establecen conexiones entre las características de superficie y las estructuras profundas. Esto lleva a que los rasgos del estereotipo se establezcan a partir de una realidad inamovible e ilegítima. Es así como los estereotipos basados en diferencias físicas pueden llegar a ser particularmente perniciosos por su rigidez y su fundamento "observable y objetivo". El estudio del impacto del esencialismo sobre los estereotipos nos permite comprender de qué manera las etiquetas arbitrarias asignadas a las diferentes categorías sociales llegan a impregnarse de un sentido profundo. Al conectar lo observado con lo inherente pueden justificarse y racionalizarse convenciones presentes en la división social (Yzerbyt, Estrada, Corneille, Seron, \& Demoulin, 2004; Yzerbyt \& Rogier, 2001). Un estudio reciente conducido por Bastian y Haslam (2006) confirma que las personas esencialistas usan más los estereotipos y explican las diferencias entre las personas mediante factores innatos.

Entre los fenómenos intergrupales clásicos en el que se estudia actualmente el efecto del esencialismo, se encuentra la evaluación y el enfrentamiento de la desviación al interior del grupo. Estudios conducidos por Marques e Yzerbyt (1988) (ver también Marques et al., 1988) mostraron que la evaluación de un miembro desviado es más dura cuando éste forma parte del propio grupo que cuando forma parte de un exogrupo. Este fenómeno ha sido llamado "el efecto de la oveja negra". Por ejemplo, si un sacerdote comete una falta que vulnera elementos centrales de su identidad social (como enamorarse), va a ser evaluado con mayor dureza por otros religiosos que por personas que no pertenecen a este grupo. Sabemos que una estrategia de autoprotección del componente social del concepto del sí mismo, consiste en conservar una diferenciación positiva entre su grupo y los otros grupos del entorno social (Tajfel, 1978). Este sesgo al exterior del grupo puede acompañarse de un sesgo endogrupal consistente en que los individuos normativos (que poseen las características deseables y contribuyen a la valorización positiva del grupo), tienen una mayor posibilidad de reforzar su positividad si "alejan" a los miembros menos deseables, acentuando su diferencia con ellos a través del aumento de su negatividad. Este efecto es susceptible de ser sensible al fenómeno del esencialismo de los grupos. Por consiguiente: el esencialismo implica, a nuestro juicio, un cierto número de consecuencias sobre la manera en la que las personas comprenden su pertenencia a un grupo. Cuando un individuo tiene una concepción esencialista de los grupos sociales, la creencia en la existencia de esencias da una cierta "objetividad" a las diferencias entre los grupos. Una de las consecuencias de esta creencia es que la pertenencia a un grupo es percibida como inalterable fijando las fronteras entre los grupos y volviéndolas impermeables.

¿Cómo enfrentan los individuos que tienen una percepción esencialista de los grupos la presencia de una "Oveja Negra”? ¿Cuáles son las estrategias que utilizan para proteger la positividad de su grupo? Investigaciones preliminares (Estrada, 2003) examinan el impacto de las creencias esencialistas en la percepción de miembros desviados.

Dos experimentos que manipulan el esencialismo muestran que aquellos que poseen este tipo de creencias tienden a obviar las diferencias observadas cuando éstas son de menor cuantía y utilizan estrategias de expulsión simbólica en comparación a aquellos miembros del grupo que manifiestan una disidencia extrema o evidente. El primer resultado es entendido como producto de la tendencia a percibir a los miembros del grupo como homogéneos y a "negar" la existencia de diferencias que no comprometen la exis- 
tencia de una esencia común. El segundo es interpretado como el resultado de la imposibilidad de expulsar a un miembro de un grupo que está cimentado en criterios "objetivos". Esta imposibilidad obliga al perceptor "esencialista" a buscar una estrategia alternativa de expulsión, que consiste en aumentar la homogeneidad entre los miembros normativos creando así una distancia entre ellos y el miembro desviado. De esta forma, la expulsión del grupo se concreta sin tener que movilizar las fronteras del grupo. Adicionalmente, se observó que los sujetos que activaron creencias esencialistas se perciben a sí mismos como más prototípicos y representativos de su grupo que aquellos sujetos que no activaron estas creencias. Este último resultado es compatible con los observados en los estudios sobre esencialismo y sus efectos sobre el proceso de influencia social en búsqueda de consenso grupal.

Dos experimentos sobre esta última cuestión (Estrada, 2003) muestran que los sujetos que han activado creencias esencialistas, se perciben como parte de una unidad relacional con su propio grupo. Como consecuencia, la búsqueda de consenso consiste simplemente en la proyección de sus propias ideas en el grupo, ya que éstas son consideradas como altamente prototípicas. Concretamente, los sujetos que trabajaron en grupos esencializados, negocian su posición consensual buscando el punto más cercano posible a cada posición individual. Los sujetos portadores de esta creencia sobre el origen de su endogrupo ven al grupo como un reflejo de sí mismos por lo que el consenso se construye a partir de las similitudes existentes. Aunque estos resultados son iniciales, parecen indicar que la percepción esencialista de los grupos cambia las dinámicas de interacción facilitando el logro de un consenso participativo, pero frena la búsqueda de soluciones innovadoras. Por ejemplo, un grupo de personas que pertenecen a un club deportivo y entienden esta pertenencia como el reflejo de una esencia profunda, al momento de enfrentar una situación de negociación donde todos los miembros del grupo expresan una parte de la esencia común, tendrán menos disposición a abandonar sus puntos de vistas originales llegando a un consenso que busca respetar la posición de cada uno, siendo entonces menos probable que obtengan conclusiones originales, fuera de las planteadas inicialmente en el grupo.

Uno de los tópicos de reciente interés en psicología social es el fenómeno de la proyección social, que fue definida por Allport (1954) como la tendencia de las personas a creer que sus pensamientos, sentimientos y comportamientos son compartidos por los otros. Más específicamente, la proyección social puede ser entendida como la tendencia general de los individuos a proyectar sus propias opiniones, actitudes y comportamientos hacia los miembros de su grupo de pertenencia. Esta definición implica que la percepción de un individuo respecto de un grupo social es determinada por la percepción que este individuo tiene de sí mismo y del grupo al que pertenece.

Una de las formas de proyección social más estudiada en psicología social es la tendencia de las personas a sobreestimar el consenso que tienen sus características o comportamientos, es decir, a percibir que aquello que son, piensan o hacen es común o altamente probable. Este fenómeno ha sido llamado "efecto de falso consenso" (Ross, Greene \& House, 1977). En una investigación conducida por Cadinu y De Amicis (1999), se observó que cuando los individuos se perciben a sí mismos y su grupo como similares, los juicios sobre ambos (individuo y endogrupo) se realizan más rápidamente y con menos errores. Los atributos del endogrupo se transforman en una parte de la imagen de sí mismo y facilitan la accesibilidad de la información sobre sí mismo. Estudios recientes desarrollados por Ames (2004) muestran que la percepción de similitud afecta de manera significativa la proyección social. En la medida en que una persona debe inferir un comportamiento respecto de otros, la percepción inicial de similitud se traduce en el uso de la información sobre sí mismo intensificando la proyección. Respecto de los aportes que el esencialismo psicológico puede dar en este campo, es necesario recordar que los individuos que poseen o han activado estas creencias, perciben la similitud de una forma particular. Cuando un individuo percibe los grupos a partir de una visión esencialista, ve las similitudes de superficie entre los miembros de un grupo como la expresión de la existencia de similitudes profundas (Yzerbyt \& Schadron, 1996). Investigaciones recientes muestran que la activación de las creencias esencialistas tiene como consecuencia la percepción de mayor similitud entre un individuo y su grupo, y que el poder inductivo de esta similitud implica que se piensa que su impacto va más allá de lo observado (actitud) y puede manifestarse en la inteligencia, el carácter moral y la personalidad. Estudios preliminares (Estrada, 2003) indican que efectivamente el esencialismo modera la proyección social hacia el endo y el exogrupo. Cuando se trata del endogrupo, los individuos esencialistas muestran una mayor 
proyección social sobre su endogrupo que aquellos que no lo son. Además, los esencialistas muestran una tendencia a mostrar proyección social negativa hacia su exogrupo. Al reconocer la existencia de similitudes con su endogrupo, reconoce al mismo tiempo las diferencias profundas con los otros grupos. Estos resultados iniciales nos parecen auspiciosos para el proceso de búsqueda de variables implicadas en la construcción y encuentro de identidades sociales (culturales, religiosas, nacionales, etc.) que pueden ser percibidas como "irreconciliables".

\section{Conclusiones}

Al revisar los avances en el estudio de las TI y, en particular, del esencialismo psicológico, podemos constatar que existe un número significativo de equipos de investigación que se encuentran realizando esfuerzos por comprender sus efectos en el terreno de las relaciones inter e intragrupo. Aunque se trata de un constructo relativamente nuevo, cuyos límites aún son imprecisos y que se encuentra en proceso de comprobación empírica, no es menos cierto que la revisión del estado actual de esta área resulta prometedora. Las razones de este interés se basan en que los estudios desarrollados durante los últimos 8 años, indican que estas ideas pueden llegar a ser herramientas conceptuales potentes para facilitar el estudio de las relaciones intragrupo, para comprender cómo afectan su percepción de la realidad, sus emociones y comportamiento.

Aunque actualmente conocemos más respecto de las funciones beneficiosas intragrupales como la protección de la autoestima y la identificación grupal (Castano, 2004), todavía existen numerosas preguntas sobre el rol que cumplen en fenómenos como la mantención de identidades sociales negativas o el enfrentamiento de la disidencia grupal. En este sentido, sería interesante investigar el papel que estas creencias juegan en problemáticas sociales emergentes en nuestro país tales como, la resistencia al cambio observada en jóvenes con conducta marginal (miembros de pandillas, por ejemplo), la reincidencia delictiva y la búsqueda de caminos para el diálogo entre grupos con ideas radicales (políticas o religiosas, por ejemplo). En el plano intergrupal se conocen más sus efectos negativos en los prejuicios y la discriminación, por lo que el desafío que subsiste es el de comprender sus posibles beneficios para las relaciones entre los grupos. En general, las TI tales como el esencialismo no han sido suficientemente estudiadas en su relación con otras variables impli- cadas en las relaciones intergrupales. Tal como lo señala Levy et al. (2006), los esfuerzos desarrollados hasta ahora han sido menores en consideración del poderoso potencial que este tipo de teorías tiene para explicar fenómenos asociados al pensamiento y a la dinámica de la interacción social.

\section{Referencias}

Allport, G. (1954). The nature of prejudice. Reading, MA: Addison Wesley.

Ames, D. (2004). Strategies for social influence: A similarity contingency model of projection and stereotyping in attribute prevalence estimates. Journal of Personality and Social Psychology, 87, 573-585.

Bacova, V. (1998). Implicit personal theories on specific domains of the social world. Studia Psychologica, 40, 255-260.

Bar-Tal, D. (1990). Causes and consequences of delegitimization: Models of conflict and ethnocentrism. Journal of Social Issues, 46, 65-81.

Bastian, B. \& Haslam, N. (2006). Psychological essentialism and stereotype endorsement. Journal of Experimental Social Psychology, 42, 228-235

Beauvois, J. L. (1984). La psychologie quotidienne. Paris: P.U.F.

Beauvois, J. L. \& Dubois, N. (1991). Internal/External orientations and psychological information processing. European Bulletin of Cognitive Psychology, 11, 193-212.

Beauvois, J. L. \& Joule, R. (1982). Dissonance versus selfperception theories: A radical conception of Festinger's theory. Journal of Social Psychology, 117, 99-113.

Bruner, J. S. \& Tagiuri, R. (1954). The perception of people. (Vol. 2). Cambridge: Addison-Wesley.

Cadinu, M. R. \& De Amicis, L. (1999). The relationship between the self and the ingroup: When having a common conception helps. Swiss Journal of Psychology, 58, 226232 .

Castano, E. (2004). In case of death, cling to the ingroup. European Journal of Social Psychology, 34, 375-384.

Chiu, C. Y., Dweck, C. S., Tong, J. Y. Y. \& Fu, J. H. Y. (1997). Implicit theories and conceptions of morality. Journal of Personality and Social Psychology, 73, 923940.

Chiu, C. Y., Hong, Y. Y. \& Dweck, C. S. (1997). Lay dispositionism and implicit theories of personality. Journal of Personality and Social Psychology, 73, 1930 .

Cronbach, L. J. (1955). Processes affecting scores on "understanding of others" and "assumed similarity". Psychological Bulletin, 52, 177-193.

Dweck, C. S., Chiu, C. Y. \& Hong, Y. Y. (1995). Implicit theories. Elaboration and extension of the model. Psychological Inquiry, 6, 322-333.

Dweck, C. S., Hong, Y. Y. \& Chiu, C. Y. (1993). Implicit theories. Individual differences in the likelihood and meaning of dispositional inference. Personality and Social Psychology Bulletin, 19, 644-656.

Estrada C. (2003). L'impact de l'essentialisme psychologique sur les processus intra- et intergroupes. Tesis de doctorado no publicada, Université catholique de Louvain, Bélgica.

Estrada, C., Yzerbyt, V. \& Seron, E. (2004). El efecto del esencialismo psicológico sobre las teorías ingenuas de las 
diferencias grupales. Psicothema, 16(2), 181-186.

Evans, E. M. (2001). Cognitive and contextual factors in the emergence of diverse belief systems: Creation versus evolution. Cognitive Psychology, 42, 217-266.

Fouts, G. \& Vaughan, K. (2002). Locus of control, television viewing, and eating disorder symptomatology in young females. Journal of Adolescence, 25, 307-311.

Furnham, A. (1990). Commonsense theories of personality. En R. Semin \& J. Kenneth (Eds.), Every understanding: Social and scientific implications (pp. 173-203). London: Sage.

Gervey, B. M., Chiu, C. Y., Hong, Y. Y. \& Dweck, C. S. (1999). Differential use of person information in decisions about guilt versus innocence: The role of implicit theories. Personality and Social Psychology Bulletin, 25, 17-27.

Gil-White, F. J. (2001). Are ethnic groups biological "species" to the human brain? Essentialism in our cognition of some social categories. Current Anthropology, 42, 515554.

Gross, C. (1961). Intrajudge consistency in rating of heterogeneos person. Journal of Abnormal and Social Psychology, 62, 605-610.

Haslam, N., Rothschild, L. \& Ernst, D. (2002). Are essentialist beliefs associated with prejudice? British Journal of Social Psychology, 41, 87-100.

Heider, F. (1958). The psychology of interpersonal relations. New York: Wiley.

Hirschfeld, L. A. (1995a). Do children have a theory of race. Cognition, 54, 209-252.

Hirschfeld, L. A. (1995b). The inheritability of identity Children's understanding of the cultural biology of race. Child Development, 66, 1418-1437.

Hirschfeld, L. A. (1998). Natural assumptions: Race, essence, and taxonomies of human kinds. Social Research, 65, 331-349.

Hirschfeld, L. A. (1999). La règle de la goutte de sang ou comment l'idée de race vient aux enfants. L'Homme, $150,15-40$.

Hollander, J. A. \& Howard, J. A. (2000). Social psychological theories on social inequalities. Social Psychology Quarterly, 63, 338-351.

Hong, Y. Y., Chiu, C. Y., Dweck, C. S., Lin, D. M. S. \& Wan, W. (1999). Implicit theories, attributions, and coping: A meaning system approach. Journal of Personality and Social Psychology, 77, 588-599.

Hong, Y. Y., Chiu, C. Y., Dweck, C. S. \& Sacks, R. (1997). Implicit theories and evaluative processes in person cognition. Journal of Experimental Social Psychology, 33, 296-323.

Hunter, D. (2002). Development of a aviation safety locus of control scale. Aviation space and environmental medicine, 73, 1184-1188.

Jerphagnon, L. (1987). Dictionnaire des grandes philosophies. Toulouse: Privat.

Kelly, G. A. (1955). The psychology of interpersonal constructs. New York: Norton \& Compagny.

Kirkcaldy, B. D., Shephard, R. J. \& Furnham, A. F. (2002). The influence of type A behaviour and locus of control upon job satisfaction and occupational health. Personality and Individual Differences, 33, 1361-1371.

Lefcourt, H. M. (1973). The functions of the illusions of control and freedom. American Psychologist, 28, 417-425.

Levy, S. R., Chi-yue, C. \& Ying-yi, H. (2006). Lay theories ant intergroup relations. Group Processes and Intergroup Relations, 9(1), 5-24.
Levy, S. R., Stroessner, S. J. \& Dweck, C. S. (1998). Stereotype formation and endorsement: The role of implicit theories. Journal of Personality and Social Psychology, 74, 14211436.

Leyens, J. P. (1983). Sommes-nous tous des psychologues? Approche psychosociale des théories implicites de personnalité. Bruxelles: Mardaga.

Leyens, J. P., Paladino, P. M., Rodriguez-Torres, R., Vaes, J., Demoulin, S. \& Rodriguez-Perez, A. (2000). The emotional side of prejudice: The attribution of secondary emotions to ingroups and outgroups. Personality and Social Psychology Review, 4, 186-197.

Leyens, J. P., Rodriguez-Perez, A., Rodriguez-Torres, R., Gaunt, R., Paladino, M. P. \& Vaes, J. (2001). Psychological essentialism and the differential attribution of uniquely human emotions to ingroups and outgroups. European Journal of Social Psychology, 31, 395-411.

Manger, T., Eikeland, O. J. \& Asbjornsen, A. (2002). Effects of social-cognitive training on student's locus of control. School Psychology International, 23, 342-354.

Markus, H. (1977). Self-schemata and processing information about the self. Journal of Personality and Social Psychology, 35, 63-78.

Marques, J. M. \& Yzerbyt, V. (1988). The black sheep effect: Judgmental extremity towards in-group members in interand intra-group situations. European Journal of Social Psychology, 18, 287-292.

Marques, J., Yzerbyt, V. \& Leyens, J. P. (1988). Extremity of judgments towards in-group members as a function of in-group identification. European Journal of Social Psychology, 18, 1-16.

Martin, C. L. \& Parker, S. (1995). Folk theories about sex and race differences. Personality and Social Psychology Bulletin, 21, 45-57.

Medin, D. L. (1989). Concepts and conceptual structure. American Psychologist, 44, 1469-1481.

Miller, R. L. \& Mulligan, R. D. (2002). Terror management: The effects of mortality salience and locus of control on risk-taking behaviors. Personality and Individual Differences, 33, 1203-1214.

Morris, M. W., Menon, T. \& Ames, D. R. (2001). Culturally conferred conceptions of agency: A key to social perception of persons, groups, and other actors. Personality and Social Psychology Review, 5, 169-182.

Myers, D. (2004). Exploraciones de la psicología social. Madrid: McGraw-Hill.

Nelkin, D. \& Lindee, S. (1995). The DNA mystique: The gene as a cultural icon. New York: W. H. Freeman.

Newcomb, T. (1931). An experiment designed to test the validity of a rating technique. Journal of Educational Psychology, 22, 279-289.

Nisbett, R., Peng, K., Choi, I. \& Norezayan, A. (2001). Culture and systems of thought: Holistic versus analytic cognition. Psychological Review, 108, 291-310

Plaks, J. E., Stroessner, S. J., Dweck, C. S. \& Sherman, J. W. (2001). Person theories and attention allocation: Preferences for stereotypic versus counterstereotypic information. Journal of Personality and Social Psychology, 80, 876-893.

Rocher, S. J. (1997). Essentialisme psychologique, théories naives et illusion de corrélation. Tesis de doctorado no publicada, Université catholique de Louvain-la-Neuve, Bélgica. 
Rodrigo, M. (1994). Etapas, contextos, dominios y teorías implícitas en el conocimiento social. En M. Rodrigo (Ed.), Contexto y desarrollo social (pp. 26-46). Madrid: Síntesis.

Rodrigo, M., Rodríguez, A. \& Marrero, J. (1993). Las teorías implicitas: Una aproximación al conocimiento cotidiano. Madrid: Visor.

Ross, L., Greene, D. \& House, P. (1977). The "false consensus effect": An egocentric bias in social perception and attribution processes. Journal of Experimental Social Psychology, 13, 301.

Rotter, J. B. (1966). Generalized expectancies for internal versus external control of reinforcement. Psychological Monographs, 80, 1-28.

Rothbart, M. \& Taylor, M. (1992). Category labels and social reality: Do we view social categories as natural kinds? En K. Semin \& A. Fiedler (Eds.), Language, interaction and social cognition (pp. 11-36). London: Sage.

Secord, P. F. \& Backman, C. W. (1974). Social psychology. New York: Mc Graw-Hill.

Sherif, M. (1948). An outline of social psychology. New York: Harper and Row.

Tajfel H. (1978). Differentiation between social groups: Studies in the social psychology of intergroup relations. London: Academic Press

Thorndike, E. L. (1920). A constant error in psychological ratings. Journal of Applied Psychology, 4, 25-29.

Vaes, J., Paladino, M. P. \& Leyens, J. P. (2002). The lost email: Prosocial reactions induced by uniquely human emotions. British Journal of Social Psychology, 41, 521534.

Wishner, J. (1960). Reanalysis of "impressions of personality". Psychological Review, 67, 112.

Yzerbyt, V., Estrada, C., Corneille, O., Seron, E. \& Demoulin, S. (2004). Subjective essentialism in action: Self anchoringand social control as consequences of fundamental social divides. En V. Yzerbyt, C. M. Judd \& O. Corneille (Eds.), The psychology of group perception: Contributions to the study of homogeneity, entitativity, and essentialism (pp. 101-124). Philadelphia: Psychology press.

Yzerbyt, V., Corneille, O. \& Estrada, C. (2001). The interplay of subjective essentialism and entitativity in the formation of stereotypes. Personality and Social Psychology Review, 5, 141-155.

Yzerbyt, V., Rocher, S. \& Schadron, G. (1997). Stereotypes as explanations: A subjective essentialistic view of group perception. En R. Spears, P. Oakes, N. Ellemers \& S. A. Haslam (Eds.), The social psychology of stereotyping and group life (pp. 20-50). Oxford: Blackwell published.

Yzerbt, V. \& Rogier, A. (2001). Blame it on the group: Entitativity, subjective essentialism and social attribution. En J. Jost \& B. major (Eds.), The psychology of legitimacy: Emerging perspectives on ideology, justice and intergroup relations. (pp. 20-49). New York: Cambridge University Press.

Yzerbyt, V. \& Schadron, G. (1996). Connaître et juger autri: Une introduction à la cognition sociale. Grenoble: Presses Universitaires.

Fecha de recepción: Octubre de 2006.

Fecha de aceptación: Enero de 2007. 